Tropical Journal of Pharmaceutical Research July 2017; 16 (7): 1455-1463

ISSN: $1596-5996$ (print); 1596-9827 (electronic)

(C) Pharmacotherapy Group, Faculty of Pharmacy, University of Benin, Benin City, 300001 Nigeria.

All rights reserved.

Available online at http://www.tjpr.org

Original Research Article

http://dx.doi.org/10.4314/tjpr.v16i7.1

\title{
Carbopol hydrogel/sorbitan monostearate-almond oil based organogel biphasic formulations: Preparation and characterization of the bigels
}

\author{
Velichka Y Andonova ${ }^{1,2 \star}$, Petya T Peneva ${ }^{1,2}$, Elisaveta G Apostolova ${ }^{3}$, Teodora \\ D Dimcheva ${ }^{4}$, Zhivko L Peychev ${ }^{4}$ and Margarita I Kassarova ${ }^{1,2}$ \\ ${ }^{1}$ Department of Pharmaceutical Sciences, Faculty of Pharmacy, Medical University - Plovdiv, ${ }^{2}$ Technological Center for \\ Emergency Medicine (TCEMED), Plovdiv, ${ }^{3}$ Department of Pharmacology and Drug Toxicology, Faculty of Pharmacy, \\ ${ }^{4}$ Department of Medical Informatics, Biostatistics and e-Learning, Faculty of Public Health, Medical University - Plovdiv, \\ Plovdiv, Bulgaria
}

*For correspondence: Email: andonova_v@abv.bg; Tel: +359888603272

Sent for review: 10 March 2017

Revised accepted: 19 June 2017

\begin{abstract}
Purpose: To obtain and evaluate carbopol hydrogel/sorbitan monostearate-almond oil-based organogel biphasic formulations (bigels) as a semi-solid vehicle for medicated topical applications.

Methods: Bigel formulations were obtained under mild conditions at a hydrogel/organogel ratio of 80/20, 70/30, and 60/40 (w/w). Their stability, viscosity, spreadability, microarchitecture, and acute skin toxicity were evaluated.

Results: Two formulations, prepared at ratios of $80 / 20$ and 70/30, were stable based on intermediate stability testing, and had a similar viscosity and spreadability $(38.0 \pm 1.0 \mathrm{~mm}$ and $37.3 \pm 0.6 \mathrm{~mm}, p>$ 0.05 , respectively). Both of these formulations had a bimodal droplet size distribution and very similar values for the droplet mean diameter $(0.33 \pm 0.05 \mu \mathrm{m}$ and $2.35 \pm 0.44$; and $0.34 \pm 0.04 \mu \mathrm{m}$ and $2.59 \pm$ $0.21 \mu \mathrm{m})$. The formulation obtained at a ratio of $60 / 40$ was unstable during storage. The in vivo results did not reveal any signs of skin toxicity.

Conclusion: Considering their beneficial properties, the developed bigels are a potential semi-solid vehicle for topical application and exhibit a moisturizing effect.
\end{abstract}

Keywords: Almond oil, Bigels, Carbopol hydrogel, Moisturizing effect, Organogel, Sorbitan monostearate

Tropical Journal of Pharmaceutical Research is indexed by Science Citation Index (SciSearch), Scopus, International Pharmaceutical Abstract, Chemical Abstracts, Embase, Index Copernicus, EBSCO, African Index Medicus, JournalSeek, Journal Citation Reports/Science Edition, Directory of Open Access Journals (DOAJ), African Journal Online, Bioline International, Open-J-Gate and Pharmacy Abstracts

\section{INTRODUCTION}

Bigels are innovative formulations that constitute two-phase structured systems obtained by mixing hydrogel and organogel. These systems can overcome the disadvantages of both types of gels, including the limited ability to cross lipophylic barriers of the skin for hydrogels and the low patient compliance for organogels due to their stickiness and oily residues [1]. They can also combine all advantages of both drug formulations: (i) the ability to accommodate both hydrophilic and lipophilic drugs; (ii) ensure topical or transdermal drug delivery; (iii) provide controlled drug delivery; and (iv) improve patient compliance [1,2]. Furthermore, Almeida et al [3] demonstrated that bigel formulations have an enhanced moisturizing effect. These properties make them very promising candidates for topical formulations.

Olive oil [2], sesame oil [4,5], sunflower oil [6], 
soybean oil $[1,7]$ and fish oil $[8,9]$ are the most commonly used oils for bigel preparation. Oleogels prepared from these oils are combined with hydrogels of natural and synthetic polymers: guar gum [4], gelatine [1], sodium alginate, hydroxypropyl methylcellulose [8], and carbopol $[5,9]$.

Almond oil has several advantageous properties $[10,11]$ and is commonly used in cosmetic products. Sorbitan monostearate (Span $\AA^{\circledR}$ 60, SMS, hydrophilic-lipophilic balance value 4.7 ) is a hydrophobic nonionic surfactant used in pharmaceutical formulations as a water-in-oil emulsifying agent. This compound gels several organic solvents, as well as a range of vegetable oils $[4,5,12]$. The aim of this study was to obtain and evaluate carbopol hydrogel/SMS-almond oil based organogel biphasic formulations (bigels) as a vehicle for semi-solid drug formulations with a topical application.

\section{EXPERIMENTAL}

\section{Materials}

All materials used in the study including Carbopol $^{\otimes} 940$ (The Lubrizol Corporation, USA), propylene glycol (Sigma-Aldrich, USA), Span ${ }^{\circledR} 60$ (Sigma-Aldrich, USA), $95 \%$ ethanol (v/v), almond oil, triethanolamine, and purified water were of pharmaceutical grade.

\section{Animals}

The in vivo experiment was approved by the Bulgarian Food Safety Agency (permit no. 88/09.01.2014) and Ethics Committee of the Medical University of Plovdiv, Bulgaria (approval no. 1/22.01.2015) [13]. The experiment was performed in compliance with the Basel Declaration [14] and ICLAS Ethical Guideline for Researchers [15].

Six male Wistar rats (weight of $180-400 \mathrm{~g}$ ) were used and maintained under standard laboratory conditions (temperature $22 \pm 1{ }^{\circ} \mathrm{C}$, humidity 45 $\%$, and 12-h light-cycle). The rodents received food and water ad libitum [13].

\section{Bigel preparation}

To prepare bigels, both phases (hydrophilic and lipophilic) were obtained separately. The hydrogel contained Carbopol ${ }^{\circledR} 9401.0 \%(\mathrm{w} / \mathrm{w})$, propylene glycol $5.0 \%(\mathrm{w} / \mathrm{w})$ [16], ethanol $95 \%$ (v/v) $10.0 \%(\mathrm{w} / \mathrm{w})$, and purified water $84.0 \%$ $(\mathrm{w} / \mathrm{w})$. The organogel contained almond oil 85.0 $\%(w / w)$ and $\operatorname{Span}^{\oplus} 6015.0 \%(w / w)$. Briefly, the preparation was prepared as follows: the weighted Carbopo ${ }^{\circledR} 940$ was dispersed in the mixture of purified water, propylene glycol, and ethanol $\left(25^{\circ} \mathrm{C}, 400 \mathrm{rpm}\right)$. A stable hydrogel was formed after adding triethanolamine $(\mathrm{pH} 5.5-$ 6.5). Span® 60 was dissolved in almond oil (60 ${ }^{\circ} \mathrm{C}, 100 \mathrm{rpm}$ ). A bigel was obtained when the heated organogel was added to the hydrogel under continuous stirring (500 rpm) to obtain a homogeneous mixture and with cooling to ambient temperatures. Three bigel formulations were prepared in hydrogel/organogel ratios of $80 / 20,70 / 30$, and $60 / 40(\mathrm{w} / \mathrm{w})$; these were named BG20, BG30, and BG40, respectively.

The prepared bigels were inspected visually for their color, homogeneity, consistency, and phase separation [7].

\section{Optical microscopy}

An optical microscope (Leica DM2000 LED, Leica Microsystems, Germany) equipped with a camera (Leica DMC 2900) and software for image processing (Leica Application Suite, LAS) was used. Samples were observed at a magnification of $40 x$ after staining with a solution of methylene blue (methylthioninium chloride) 1.5 $\%(w / v)$.

\section{Stability study of the bigel formulations}

Intermediate stability studies of the three bigel formulations were prepared according to the $\mathrm{ICH}$ guidelines. Bigels were packed in tightly closed plastic containers and stored at $30 \pm 2{ }^{\circ} \mathrm{C} / 65 \pm 5$ $\% \mathrm{RH}$ for 6 months [17]. Their physical stability was evaluated through observation for any change in color, phase separation, or syneresis at regular time intervals $[5,18]$. To assess changes in their microarchitecture during storage, they were subjected to optical microscopy, as described above.

\section{Droplet size distribution}

Droplet size distribution analysis of the tested formulations was performed using a Nanotrac Wave apparatus (Microtrac, Inc., USA). Microtrac software automatically provided information on multi-modal distributions. The samples were prepared using an equal quantity of bigels (1\% $\mathrm{w} / \mathrm{w}$ ) in phosphate buffer at $\mathrm{pH}$ 5.5. They were subjected to ultrasonic impact for $10 \mathrm{~min}$ to obtain a homogeneous emulsion before measuring the droplet mean diameter. Samples with a volume of $3 \mathrm{~mL}$ were analyzed at $25.0 \pm$ $0.5^{\circ} \mathrm{C}$. The measurements were performed three times and the results were recorded as the mean \pm standard deviation (SD) [5]. 


\section{Determination of $\mathrm{pH}$}

The $\mathrm{pH}$ values of $1 \%$ aqueous dispersion of the prepared bigels were measured using a $\mathrm{pH}$ meter (inoLab pH 720), which was calibrated with standard buffer solutions. The measurement was performed three times and the $\mathrm{pH}$ of every bigel formulation was recorded as the mean value \pm SD.

\section{Determination of viscosity}

Determination of the viscosity of bigel formulations was performed using a Selecta STS-2011 viscometer (J. P. SELECTA, s. a., Spain) at $25.0 \pm 0.5^{\circ} \mathrm{C}$. A spindle $\mathrm{R} 6$ was used at $1,2,3,4,5,6$, and $10 \mathrm{rpm}$. The results were plotted after triplicate measurements (mean \pm $\mathrm{SD})$.

\section{Determination of the spreadability of bigel formulations}

Spreadability of the bigel formulations was evaluated as follows: $1 \pm 0.01 \mathrm{~g}$ of sample was pressed between two horizontal plates $(20 \times 20$ $\mathrm{cm})$, where the upper plate weighed $125 \pm 1 \mathrm{~g}$. The study temperature was maintained at $25.0 \pm$ $0.5^{\circ} \mathrm{C}$ and the bigels were evaluated $48 \mathrm{~h}$ after their preparation [19]. The spread diameter $(\phi)$ was measured after 1 min [19-21]. The measurements were performed three times and the results were recorded as the mean $\pm \mathrm{SD}$ [5].

\section{ATR-FTIR analysis}

ATR-FTIR analysis was performed with a NicoletTM iS TM 10 FT-IR Spectrometer equipped with a Smart iTRTM attenuated total reflectance sampling (ZnSe crystal) accessory (Thermo Scientific, Thermo Fisher Scientific, Inc., USA). The spectra were recorded from 4000 to $650 \mathrm{~cm}^{-1}$ using a DTGS detector. All spectra were corrected for $\mathrm{CO}_{2}$ using internal software.

\section{Acute skin toxicity testing}

Six rats (weight $250-400 \mathrm{~g}$ ) were selected for the skin toxicity tests $(n=6)$. Animals were anesthetized with an intraperitoneal injection of ketamine $(90 \mathrm{mg} / \mathrm{kgbw})$ and xylazine $(10 \mathrm{mg} / \mathrm{kgbw})$. Fur on the back of the rats was shaved and two fields with an area of $1 \mathrm{~cm}^{2}$ were marked. The sites were chosen to be as close to the rat neck as possible to avoid ingestion through animal licking of the site after treatment. One field was left untreated and one milliliter of the tested formulation was rubbed gently on the skin at the other site. The animals were returned to their cages and observed for symptoms of local and systemic toxicity. Skin toxicity was evaluated on the $1^{\text {st }}, 24^{\text {th }}$, and $48^{\text {th }}$ hour after application. The results were photographed and irritation scores were determined using a modified Draize scoring system [22].

\section{Statistical analysis}

Statistical analysis was performed using a oneway ANOVA with GraphPad InStat software 3.10 version [23]; $p<0.05$ was considered to indicate significance.

\section{RESULTS}

\section{Characteristics of bigel formulations}

The gel formation was confirmed based on the tube inversion test [5] (Figure 1). BG40 had a different structure and consistency from that of BG20 and BG30, possibly due to the greater amount of almond oil organogel and the technological conditions (temperature, stirring speed, stirring time, etc.) used in its preparation. All bigel formulations had a white color with a creamy appearance and a pleasant scent of almond oil. BG20 and BG30 were not greasy to the touch, unlike BG40, in which the oil droplets could be felt, although there was no phase separation.

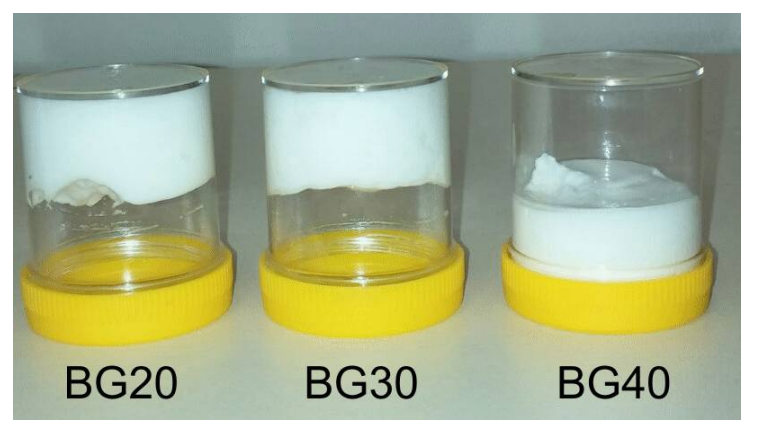

Figure 1: Bigel formulations obtained after mixing of carbopol hydrogel and almond oil organogel

\section{Optical properties}

Figure 2 presents optical micrographs of the three bigel formulations in terms of the mutual disposition of the phases. In BG20 and BG30 (Figure $2 a$ and $b$ ), the almond oil organogel is dispersed in the form of small gel droplets with regular or oval shapes into the carbopol hydrogel. The oval shape is due to spreading of the bigel formulation on the slide prior to microscopy analysis. Both micrographs show individual droplets with dimensions larger than 5 


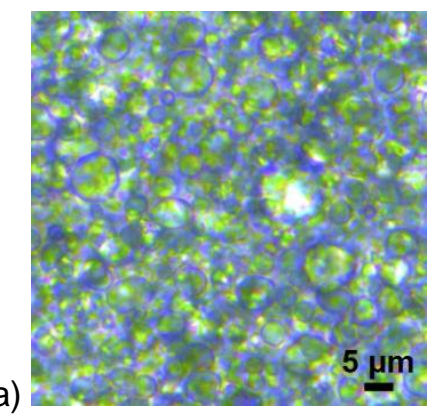

b)

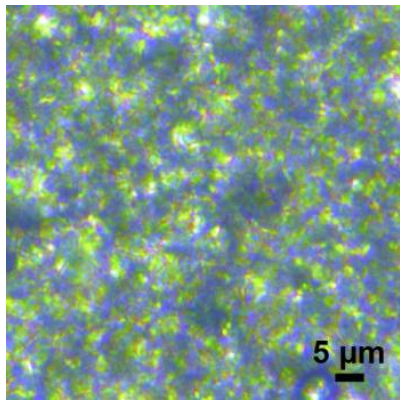

c)

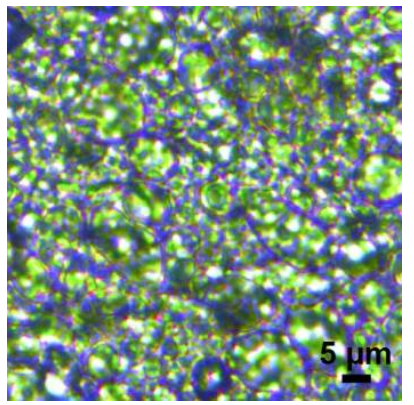

Figure 2: Optical microscopy of bigels at 40x: a) BG20; b) BG30, c) BG40 (Leica DMC2900)

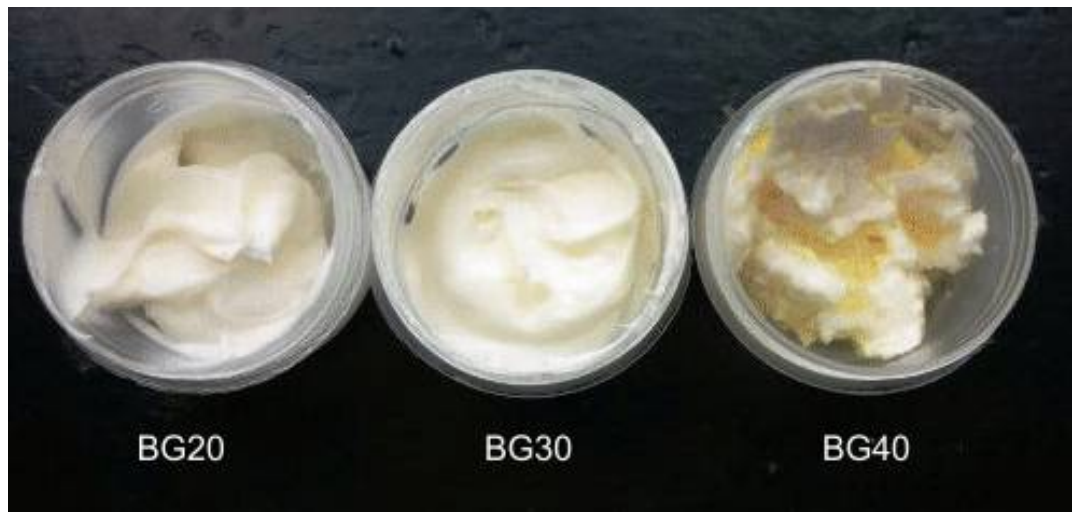

Figure 3: Three bigel formulations after 6 months of storage at $30 \pm 2{ }^{\circ} \mathrm{C}$ and $65 \% \pm 5 \%$ humidity

a)

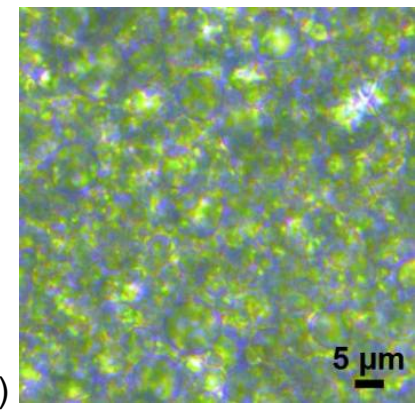

b)

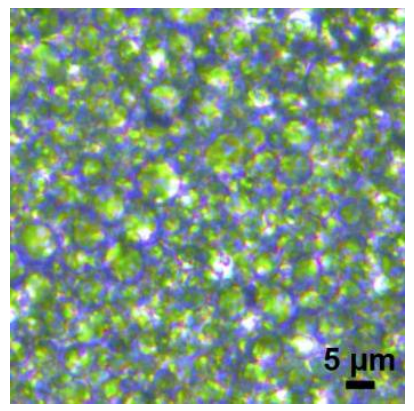

c)

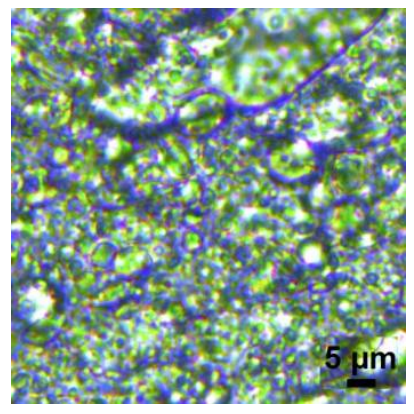

Figure 4: Optical microscopy of bigels at $40 \mathrm{x}$ after 6 months of storage at $30 \pm 2{ }^{\circ} \mathrm{C}$ and $65 \% \pm 5 \%$ humidity: a) BG20; b) BG30, c) BG40 (Leica DMC2900)

$\mu \mathrm{m}$. This microstructure of bigel formulations can provide more stability during storage. In contrast, BG40 is characterized by a non-uniform structure due to the presence of large quantities of poorly dispersed almond oil organogel, which is not homogeneously mixed with carbopol hydrogel.I

\section{Stability study of the bigel formulations}

Figure 3 shows three bigel formulations after 6 months of storage at $30 \pm 2{ }^{\circ} \mathrm{C} / 65 \% \pm 5 \% \mathrm{RH}$ [17]. No changes were observed in the physical stability of BG20 and BG30, as opposed to BG40, during the stability study. Initial changes regarding the phase separation of BG40 were observed early in the experiment after about 1 month storage, after which syneresis occurred.
Optical microscopy was performed on the three bigel formulations to examine changes in their microarchitecture during storage. Figure 4 presents the optical micrographs of BG20, BG30, and BG40 at the end of the intermediate stability study. BG20 and BG30 are characterized by a homogeneous distribution without any areas of confluence of almond oil organogel or carbopol hydrogel. This microstructure plays a crucial role in the physical stability of both bigels (BG20 and $B G 30$ ). Figure $4 \mathrm{c}$ shows the microstructure of BG40, which is characterized by a fragmented and inhomogeneous structure and large, clearly delineated vacuoles indicative of syneresis. Due to the physical instability of BG40, subsequent investigations were performed using only bigel formulations of BG20 and BG30. 

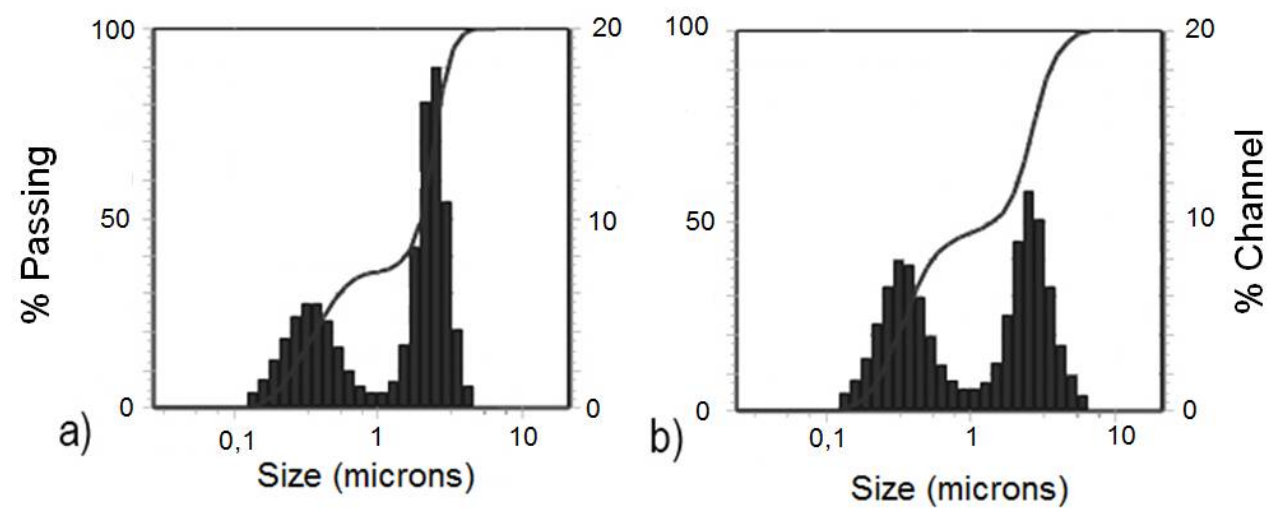

Figure 5: Droplet size distribution of the bigel formulations: a) BG20 and b) BG30

\section{Droplet size distribution}

This analysis revealed a broad bimodal droplet size distribution for both BG20 and BG30 (Figure 5). In formulation BG20, $90 \%$ of droplets were larger than $0.257 \pm 0.06 \mu \mathrm{m}$, and only $5 \%$ were larger than $3.29 \pm 0.51 \mu \mathrm{m}$. Similar results were observed for BG30. The droplet size ranged from $0.244 \pm 0.04$ to $4.06 \pm 0.62 \mu \mathrm{m}$. Table 1 shows the peak summary for the bimodal droplet size distribution of the tested formulations. The results indicated that the droplet mean diameter (the 50 $\%$ point of each mode reported) was similar in both formulations. A difference in the volume \% (the calculated contribution in percent of each peak to the total volume of the distribution) and width (a measure of the broadness of the peak under consideration) was established. Furthermore, the droplet mean diameter established for BG20 and BG30 was in agreement with the results of the optical microscopy analysis, as presented above.

\section{$\mathrm{pH}$ of bigel formulations}

The $\mathrm{pH}$ values of the bigel formulations were $5.66 \pm 0.03$ and $6.33 \pm 0.02$ for BG20 and BG30, respectively. These values, which are within the range of the physiological $\mathrm{pH}$ of healthy skin, are important for physiological tolerance and a possible lack of skin irritation in topical applications [24].

\section{Viscosity of bigel formulations}

Figure 6 shows the effect of shearing on the viscosity of bigels BG20 and BG30. Both bigel formulations exhibited pseudoplastic flow. A lower viscosity of BG20 was established compared to the viscosity of BG30 at all shearing values. An increase in shearing rate led to a decreased difference between the viscosities of both bigels. For example, the viscosities of bigel formulations were $\sim 524.7 \pm 66$ Pa.s and $613.5 \pm$ 52 Pa.s for BG20 and BG30 at 1 rpm; $122.7 \pm 17$ Pa.s and $150.7 \pm 12$ Pa.s for BG20 and BG30 at $5 \mathrm{rpm}$; and $67.1 \pm 9$ Pa.s and $84.1 \pm 5$ Pa.s for BG20 and BG30, respectively, at $10 \mathrm{rpm}$. Statistical processing of the results suggest that the difference is not statistically significant $(p>$ 0.05 ). The higher viscosity of BG30 compared to BG20 can be explained by the higher SMS content in BG30. These results confirm the findings established by Ibrachim et al [7].

\section{Determination of the spreadability of bigel formulations}

Spreadability is an important characteristic of semi-solid dosage forms: it determines extrudability from the package, the method of application (easy or difficult), accurate dosing, and patient preference. In this study, the spreadability of bigel formulations was determined by measuring the spread diameter $(\phi)$.

Table 1: Peaks of bimodal droplet size distribution of formulations for BG20 and BG30 $(n=3)$

\begin{tabular}{lcccccc}
\hline Peak & \multicolumn{3}{c}{ BG20 } & \multicolumn{3}{c}{ BG30 } \\
\cline { 2 - 7 } & $\begin{array}{c}\text { Diameter } \\
(\boldsymbol{\mu m})\end{array}$ & $\begin{array}{c}\text { Volume } \\
(\%)\end{array}$ & Width & $\begin{array}{c}\text { Diameter } \\
(\boldsymbol{\mu m})\end{array}$ & $\begin{array}{c}\text { Volume } \\
(\%)\end{array}$ & Width \\
\hline Peak 1 & $2.35 \pm 0.44$ & $64.2 \pm 7.3$ & $1.15 \pm 0.50$ & $2.59 \pm 0.21$ & $53.4 \pm 5.5$ & $1.76 \pm 0.34$ \\
Peak 2 & $0.33 \pm 0.05$ & $35.8 \pm 7.3$ & $0.30 \pm 0.06$ & $0.34 \pm 0.04$ & $46.6 \pm 5.5$ & $0.29 \pm 0.07$ \\
\hline
\end{tabular}




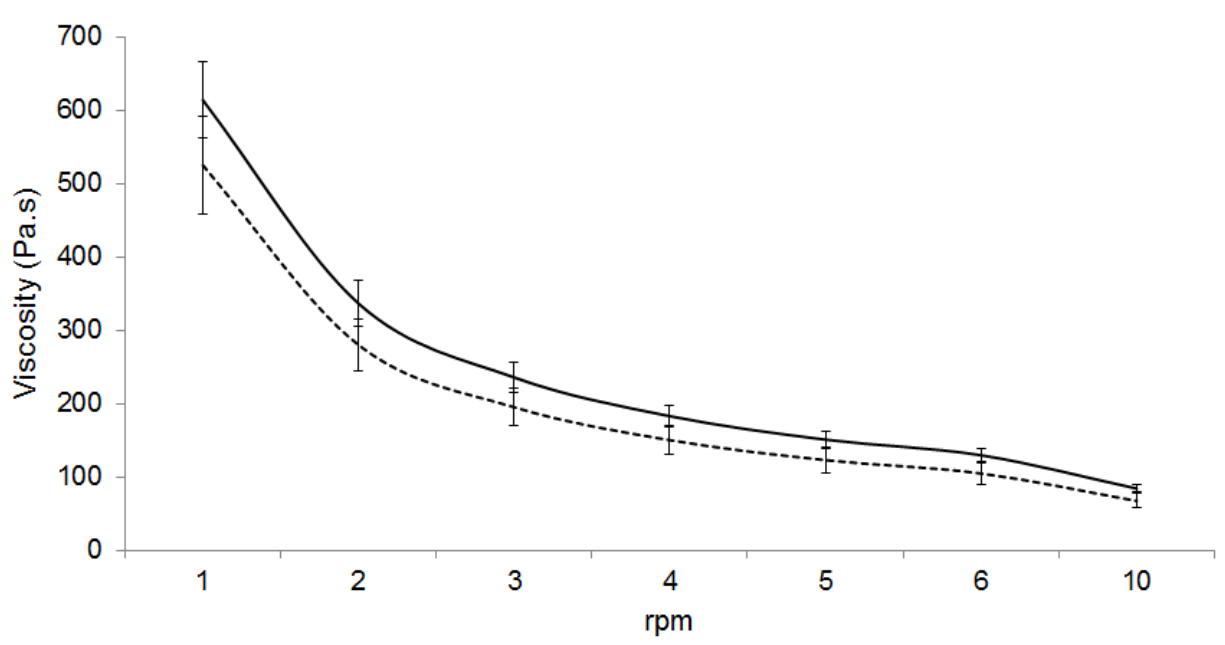

Figure 6: Effect of shearing on the viscosity of bigel formulations BG20 and BG30. Note: $\cdots \cdots . . .=B G 20 ;-=$ BG30

The diameters of resulting circles $(\phi)$ were $38.0 \pm$ $1.0 \mathrm{~mm}$ and $37.3 \pm 0.6 \mathrm{~mm}$ for BG20 and BG30, respectively. In a study on various oil-in-water creams and gels, Arvouet-Grand et al [20] and later Lardy et al [21] introduce the terms 'semistiff' and 'semi-fluid' to evaluate the spreadability of creams and gels. According to both classifications, BG20 and BG30 are semi-stiff formulations, because the established $(\phi)$ was $\leq$ $50 \mathrm{~mm}$. Based on these results, the difference between the hydrogel/organogel ratio in both bigel formulations affects the spreadability of $\mathrm{BG} 20$ and BG30 ( $p>0.05)$.

\section{ATR-FTIR spectra}

ATR-FTIR analysis was used to test for any chemical or physical interactions between the two phases: almond oil organogel and carbopol hydrogel. Figure 7 presents the spectra of bigel formulations (BG20 and BG30) and those of both gels before mixing (almond oil organogel and carbopol hydrogel).

Almond oil organogel consists of almond oil and SMS. Its characteristic absorption peaks in the range $2850-3000 \mathrm{~cm}^{-1}$, which is associated with the $\left(-\mathrm{CH}_{2}-\right)$ group, which is strong and present in the spectra of both SMS [25] and almond oil [26]; the narrow peak located at $1744.51 \mathrm{~cm}^{-1}$ is associated with the $(=\mathrm{C}=\mathrm{O})$ group [27] and the peak at $1466.21 \mathrm{~cm}^{-1}$ belongs to the $\left(-\mathrm{CH}_{2}-\right)$ group related to the $\left(-\mathrm{CH}_{3}-\right)$ group [27]. In the spectra of both bigel formulations, the peaks at 2921.29 and $2851.58 \mathrm{~cm}^{-1}$ were slightly shifted to 2917.86 and 2850.03 and $2917.72 \mathrm{~cm}^{-1}$ and $2850.16 \mathrm{~cm}^{-1}$ for BG20 and BG30, respectively. They were characterized based on the low intensity, which increased with increasing amounts of almond organogel. The same tendency was observed in terms of the peak localized at $1744.51 \mathrm{~cm}^{-1}$ in both spectra of BG20 and BG30.

The carbopol hydrogel consists of Carbopol ${ }^{B}$ 940, propylene glycol, ethanol, purified water, and triethanolamine. This composition is responsible for the following characteristic peaks in the carbopol hydrogel spectrum: the peak located at $3362.03 \mathrm{~cm}^{-1}$ is associated with free ($\mathrm{OH})$ groups; the carbonyl $(=\mathrm{C}=\mathrm{O})$ group can be determined between 1670 and $1820 \mathrm{~cm}^{-1}$, but conjugation shifts absorptions to lower wave numbers (the peak at $1644.75 \mathrm{~cm}^{-1}$ ); and peaks located at $1044.88 \mathrm{~cm}^{-1}$ indicate stretching vibration of the ethereal $\mathrm{C}-\mathrm{O}-\mathrm{C}$ group $[27,28]$. All three peaks are clearly visible in the spectra of bigel formulations of BG20 and BG30.

These results are suggestive of a lack of chemical interaction between the almond oil organogel and carbopol hydrogel, as well as the existence of a physical mixture of the two phases [5].

\section{Acute skin toxicity}

The acute skin toxicity test was conducted on six rats with BG30 to reduce the number of experimental animals used and because SMS is present in the bigel. No symptoms of skin toxicity (redness, edema), irritation, or inflammation were observed for the first $48 \mathrm{~h}$ after treatment. These results indicate that the tested BG30 formulation can be considered safe for dermal use. Our results are consistent with those of Rehman et al [29], who found that bigel formulations did not cause sensitization and may reduce imiquimod (a chemotherapeutic agent) inflammation. 


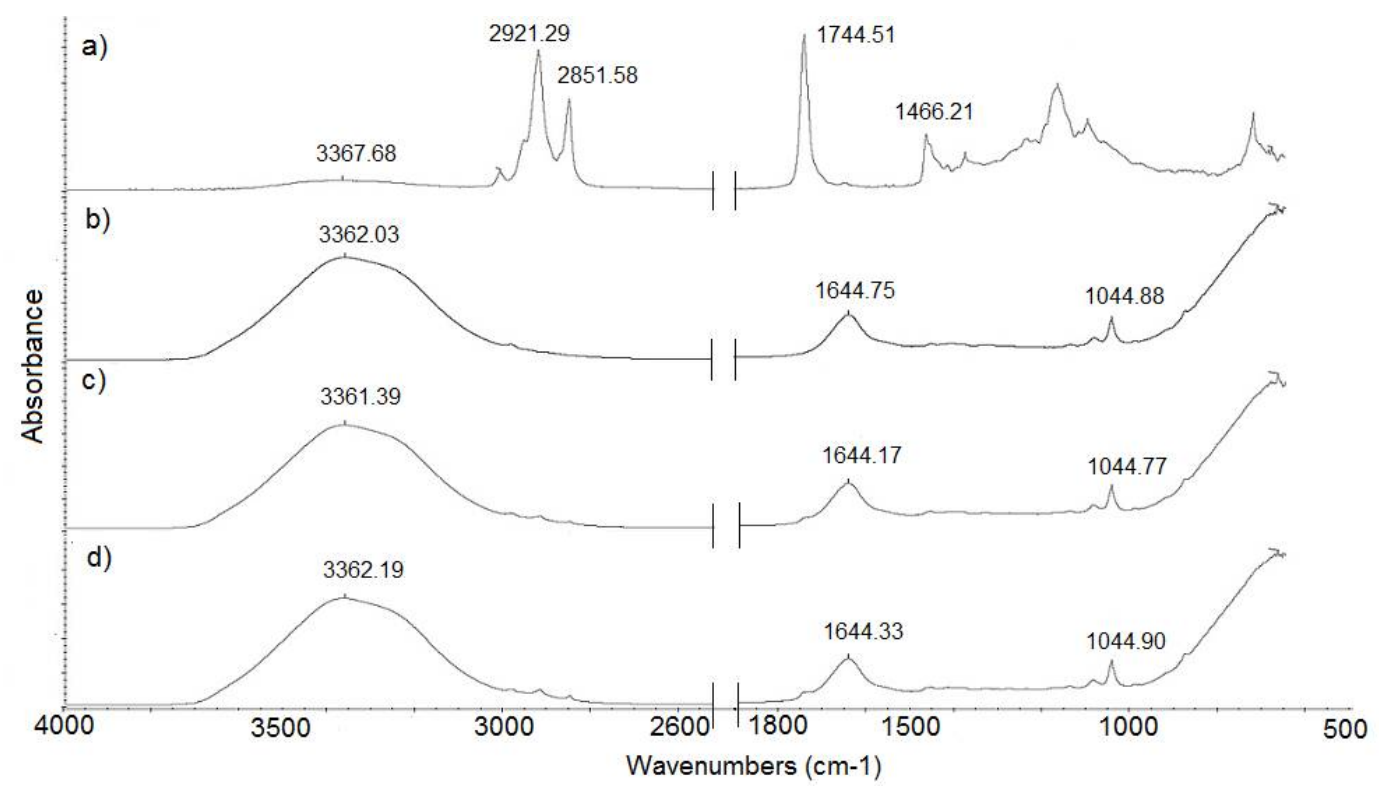

Figure 7: ATR-FTIR spectra of both bigel formulations BG20 and BG30 compared with the spectra of almond oil organogel and carbopol hydrogel: a) almond oil organogel, b) carbopol hydrogel, c) BG20, and d) BG30

\section{DISCUSSION}

Three bigel formulations with different carbopol hydrogel/almond oil organogel ratios were developed in the current study. Two formulations, BG20 and BG30, prepared at ratios of $80 / 20$ and $70 / 30$, were stable according to the intermediate stability testing, and they had similar viscosity and spreadability. We observed a bimodal droplet size distribution and similar values for the droplet mean diameter.

Regardless of the broad droplet distribution determined for both bigels, stability was maintained. This may be because most droplets were in the nanometer size range. These results were confirmed based on optical microscopy analysis, as discussed above. Furthermore, BG20 and BG30 constitute a physical mixture of carbopol hydrogel and almond oil organogel (which improves skin tolerance), which was confirmed based on acute skin toxicity testing and in accordance with the $\mathrm{pH}$ of the formulations. If a chemical interaction between the components of the bigels was observed, it would have been a prerequisite for skin sensitivity and for a change in the physical and mechanical characteristics of the system, which would affect stability and behavior as a drug delivery system. Both bigel formulations have similar characteristics; they may be used as drug delivery systems for hydrophilic and hydrophobic drugs, and selection of the bigel will depend on the targeted biopharmaceutical characteristics.

Formulation BG40, which contains $40 \%(\mathrm{w} / \mathrm{w})$ almond oil organogel, is of particular interest.
This system is very unstable and the first signs of physical instability were observed early. Syneresis was observed in the almond oil organogel as part of the bigel composition. The syneresis in BG40 can be explained by the imbalanced interfacial tension between almond oil, SMS, and the gravitational force [30].

In contrast to the results of our study, Singh et al [4] found that bigels with a higher proportion of organogel remained stable at high temperatures and maintained their structural organization. According to the authors, the long alkyl hydrocarbon chain of SMS may be the stabilizing factor in this formulation.

Molecules of the gelator tend to self-assemble via nucleation, when the organogelator-solvent melt is supersaturated (e.g., the temperature is below the melting point of the organogelator; 53 ${ }^{\circ} \mathrm{C}$ for SMS). The growth is preferentially onedimensional, which leads to the formation of fibers. The degree of supersaturation is the primary factor influencing nucleation and growth. The stable state of organogels depends on: (i) hydrogen bonding; (ii) van der Waals forces as transient forces; (iii) and the mechanism of fiber growth, which is influenced by the nature of the liquid portion of the gel [30]. The presence of almond oil used in the current study may explain the difference in the physical stability of BG40 and the bigel obtained by Singh et al [4], along with some technological differences in the preparation and storage of bigels. Rogers et al [30] found that differences in the physicomechanical characteristics of the examined organogels can be explained by the fiber growth model, as well as the storage temperature. 
However, further studies are required to explore the physical instability of BG40.

\section{CONCLUSION}

An effective method for the development of novel carbopol hydrogel/sorbitan monostearate-almond oil based bigels has been developed. The bigel formulations obtained at hydrogel/organogel ratios of $80 / 20$ and $70 / 30(\mathrm{w} / \mathrm{w})$ are characterized by a homogeneous microstructure, viscosity and spreadability that are appropriate for semi-solid formulations. The formulations did not show any signs of skin toxicity. Thus, the developed bigels appear to be suitable semi-solid vehicles for topical applications, as well as good skin moisturizers.

\section{DECLARATIONS}

\section{Acknowledgement}

The authors would like to thank all teaching and non-teaching staff of Medical University, Plovdiv, Bulgaria for their encouragement during the study.

\section{Conflict of Interest}

No conflict of interest associated with this work.

\section{Contribution of Authors}

The authors declare that this work was done by the authors named in this article and all liabilities pertaining to claims relating to the content of this article will be borne by them.

\section{Open Access}

This is an Open Access article that uses a funding model which does not charge readers or their institutions for access and distributed under the terms of the Creative Commons Attribution License (http://creativecommons.org/licenses/by/ 4.0) and the Budapest Open Access Initiative (http://www.budapestopenaccessinitiative.org/rea d), which permit unrestricted use, distribution, and reproduction in any medium, provided the original work is properly credited.

\section{REFERENCES}

1. Sagiri SS, Singh VK, Kulanthaivel S, Banerjee I, Basak $P$, Battachrya MK, Pal K. Stearate organogel-gelatin hydrogel based bigels: Physicochemical, thermal, mechanical characterizations and in vitro drug delivery applications. J Mech Behav Biomed Mater 2015; 43: 117.
2. Lupi FR, Gentile L, Gabriele D, Mazzulla S, Baldino N, de Cindio B. Olive oil and hyperthermal water bigels for cosmetic uses. J Colloid Interface Sci 2015; 459: 70-78.

3. Almeida IF, Fernandes AR, Fernandes L, Pena Ferreira MR, Costa PC, Bahia MF. Moisturizing effect of oleogel/hydrogel mixtures. Pharm Dev Technol 2008; 13: 487-494.

4. Singh VK, Banerjee I, Agarwal T, Pramanik K, Bhattacharya MK, Pal K. Guar gum and sesame oil based novel bigels for controlled drug delivery. Colloids Surf B Biointerfaces 2014; 123: 582-592.

5. Singh VK, Anis A, Banerjee I, Pramanik K, Bhattacharya MK, Pal K. Preparation and characterization of novel carbopol based bigels for topical delivery of metronidazole for the treatment of bacterial vaginosis. Mater Sci Eng C Mater Biol Appl 2014; 44: 151-158.

6. Behera B, Singh VK, Kulantaivel S, Bhattacharya MK, Paramanik K. Physical and mechanical properties of sunflower oil and synthetic polymers based bigels for the delivery of nitroimidazole antibiotic - A therapeutic approach for controlled drug delivery. Eur Polym J 2015; 64: 253-264.

7. Ibrachim MM, Hafez SA, Mahdy MM. Organogels, hydrogels and bigels as transdermal delivery systems for diltiazem hydrochloride. AJPS 2013; 8: 48-57.

8. Rehman K, Mohd Amin MC, Zulfakar MH. Development and physical characterization of polymer-fish oil bigel (hydrogel/oleogel) system as a transdermal drug delivery vehicle. J Oleo Sci 2014; 63: 961-970.

9. Rehman K, Zulfakar MH. Novel fish oil-based bigel system for controlled drug delivery and its influence on immunomodulatory activity of imiquimod against skin cancer. Pharm Res 2016; DOI: 10.1007/s11095-0162036-8.

10. Ahmad Z. The uses and properties of almond oil. Complement Ther Clin Pract 2010; 16(1): 10-12.

11. Venkatachalam M, Sathe SK. Chemical composition of selected edible nut seeds. J Agric Food Chem 2006; 54 : 4705-4714.

12. Dinte E, Tomuţă I, lovanov Ri, Leucuţa Se. Design and formulation of buccal mucoadhesive preparation based on sorbitan monostearate oleogel. Farmacia 2013; 61: 284-297.

13. Zagorchev P, Apostolova E, Kokova V, Peychev $L$. Activation of KCNQ channels located on the skeletal muscle membrane by retigabine and its influence on the maximal muscle force in rat muscle strips. Naunyn Schmiedebergs Arch Pharmacol 2016; 389: 439-446.

14. The Basel Declaration [cited 2017 Apr 18]. Available from: http://www.basel-declaration.org/basel-declaration/

15. ICLAS Ethics and Animal Welfare Committee. ICLAS Ethical Guideline for Researchers [cited 2017 Apr 18]. Available from: http://iclas.org/committees/ethics-andanimal-welfare-committee

16. Chiang C-C, Lin T-C, Chen R. Compositions and methods for the transdermal delivery of pharmaceutical compounds. Patent no 20090297591 [cited 2017 Apr 
18]. Available from: http://www.patentsencyclopedia. com/app/20090297591\#ixzz4eayG7TOR

17. Gohel MC, Patel TM. Compatibility study of quetiapine fumarate with widely used sustained release excipients. J Therm Anal Calorim 2013; 111: 2103-2108.

18. Khunt DM, Mishra AD, Shah DR. Formulation Design and Development of Piroxicam Emulgel. Int J PharmTech Res 2012; 4: 1332-1344.

19. Garg A, Aggarwal D, Garg S, Singla AK. Spreading of semisolid formulations: An update. Pharmaceutical Technology 2002; Sept: 84-105 [cited 2017 Apr 18]. Available from: http://images.alfresco.advanstar.com/ alfresco_images/pharma/2014/08/22/c0e3f115-2d3047c3-8a80-c478037fd1cf/article-30365.pdf

20. Arvouet-Grand A, Vennat B, Lejeune B, Pourrat $A$. Formulation of propolis extract emulsions part $I: \quad o / w$ creams based on nonionic surfactants and various consistency agents. Drug Dev Ind Pharm 1995; 21 : 1907-1915.

21. Lardy F, Vennat B, Pouget MP, Pourrat A. Functionalization of hydrocolloids: Principal component analysis applied to the study of correlations between parameters describing the consistency of hydrogels. Drug Dev Ind Pharm 2000; 26: 715-721.

22. Widera G, Johnson J, Kim L, Libiran L, Nyam K, Daddona PE, Cormier M. Effect of delivery parameters on immunization to ovalbumin following intracutaneous administration by a coated microneedle array patch system. Vaccine 2006; 24: 1653-1664.
23. Rai S, Paliwal R, Vaidya B, Khatri K, Goyal AK, Gupta $P N$, Vyas SP. Targeted delivery of doxorubicin via estrone-appended liposomes. J Drug Target 2008; 16 : 455-463.

24. Worth AP, Cronin MTD. The use of $\mathrm{pH}$ measurements to predict the potential of chemicals to cause acute dermal and ocular toxicity. Toxicology 2001; 169: 119-131.

25. van Lierop B, Castle L, Feigenbaum A, Boenke $A$. Spectra for the Identification of Additives in Food Packaging. Netherlands: Springer Netherlands; 1998. $p$. 441-445.

26. Yildirim K, Kostem AM. A technical glance on some cosmetic oils. ESJ 2014; 2: 425-435.

27. Silverstein, Robert; Webster, Francis; Kiemle, David. Spectrometric identification of organic compounds. 7th ed. New York: John Wiley and Sons; 2005; pp 72-126.

28. Sahoo S, Chakraborti CK, Mishra SC, Naik s, Nanda UN. FTIR and raman spectroscopy as a tool for analyzing sustained release hydrogel of ciprofloxacin/carbopol polymer. IJPSR 2011; 7: 268-277.

29. Rehman K, Aluwib MFFM, Rullahb K, Waib LK, Amina MCIM, Zulfakara MH. Probing the effects of fish oil on the delivery and inflammation-inducing potential of imiquimod. Int J Pharm 2015; 490: 131-141.

30. Rogers MA, Wright AJ, Marangoni AG. Engineering the oil binding capacity and crystallinity of self-assembled fibrillar networks of 12-hydroxystearic acid in edible oils. Soft Matter 2008; 4: 1483-1492. 